Cancer J. 2012 ; 18(2): 192-202. doi:10.1097/PPO.0b013e31824f118b.

\title{
Adjuvant Therapy for Melanoma
}

\author{
Diwakar Davar, MD, \\ Division of General Internal Medicine, University of Pittsburgh Medical Center, 200 Lothrop \\ Street, Pittsburgh, PA 15213
}

Ahmad A. Tarhini, MD, PhD, and

Division of Hematology-Oncology, University of Pittsburgh Medical Center, 5150 Centre Avenue, Pittsburgh, PA 15232, tarhiniaa@upmc.edu

\section{John M. Kirkwood, MD}

Division of Hematology-Oncology, University of Pittsburgh Medical Center, 5150 Centre Avenue,

Pittsburgh, PA 15232, kirkwoodjm@upmc.edu, Phone: 412-623-7707, Fax: 412-623-7704

\section{Abstract}

Estimates from the U.S. Surveillance, Epidemiology, and End Results (SEER) registry suggest that melanoma incidence will reach 70,230 in 2011, of which 8,790 will die. The rising incidence and predilection for young individuals makes this tumor a leading source of lost productive years in the society.

High-dose interferon- $a 2 b$ is the only agent approved for adjuvant therapy of melanoma; the improvement in relapse-free survival has been observed across nearly all published studies and meta-analyses. However toxicity affects compliance and current research is focusing upon biomarkers that may allow selection of patients with greater likelihood of response, and exploring new agents either singly or in combination that may improve upon the benefit of IFN.

In this article, we review the data for the adjuvant therapy of malignant melanoma - focusing on the results obtained with various regimens testing the several formulations of interferon-a2, and the adjuvant studies of vaccines and radiotherapy. Recent advances in the treatment of metastatic disease have established a role for CTLA-4 blockade and BRAF-inhibition, and raising hopes that these agents may have a role in the adjuvant setting. At present, several trials investigating combinations of novel agents with existing immunomodulators are underway.

\section{Introduction}

Melanoma is a disease of increasing incidence that exacts a disproportionate toll amongst the young in the population. SEER statistics suggest that of the estimated 70,230 with incident melanoma in $2011,58.5 \%$ of patients will be below the age of 64 [1].

Patients with locally advanced disease have a relatively high risk of recurrence and death despite surgery. At present, the only Food and Drug Administration (FDA)-approved adjuvant treatment option for patients with high-risk disease (primary tumor thickness of

Correspondence to: John M. Kirkwood.

Disclosures

The authors report no competing interests concerning the validity of research or for financial gain.

Conflicts of Interest

Dr. Kirkwood is a member of medical advisory boards for Genentech (a member of the Roche Group) and Merck, and a consultant for GSKBio.

Drs. Davar and Tarhini report no conflicts of interest. 
$4 \mathrm{~mm}$ or greater (T4 lesions) and/or regional lymph node metastases) is interferon- $\mathrm{a} 2 \mathrm{~b}$ (IFN$a 2 b)$.

In this article, we delineate the clinical prognostic factors that portend a heightened risk of recurrence and outline the development of IFN- $a$ in the adjuvant setting with a focus on the various clinical trials that led to the adoption of high-dose interferon (HDI) as the standard adjuvant therapy for this disease. We discuss other evolving options including vaccines, CTLA-4 blockade, chemotherapy and radiotherapy--which have yet to demonstrate reproducible survival benefits in randomized phase III trials and hence remain experimental at this time.

With the recent FDA approval of ipilimumab (Yervoy $®$ ) and vemurafenib (Zelboraf ${ }^{\circledR}$ ) for the treatment of metastatic melanoma, a once stagnant field has been rejuvenated [2-4].

Work is already underway utilizing these agents in the adjuvant setting. This article updates prior adjuvant reviews [5-6] and meta-analyses [7-10].

\section{Materials and Methods}

\section{Search Strategy and Selection Criteria}

A systematic search strategy was performed utilizing the MEDLINE, EMBASE, Cancerlit, Cochrane, ISI and Web of Science databases for articles published between January 1, 2002, and November 1, 2011. MeSH headings used included "melanoma, advanced"; and "melanoma, adjuvant" or "melanoma, interferon".

\section{Discussion}

\section{Clinical Prognostic Factors in Malignant Melanoma}

Major prognostic features associated with an increased risk of recurrence and mortality in the American Joint Committee on Cancer (AJCC) Melanoma Staging Database have been incorporated into the revised melanoma staging manual of the AJCC and International Union against Cancer (UICC) [11].

At the level of the primary tumor, three factors are critical: primary tumor thickness (Breslow's), ulceration and mitotic rate. Primary tumor thickness remains the single most important factor with 5- and 10- year survival rates declining commensurate with increasing tumor depth: 10-year survival in patients with $\mathrm{T} 1 \mathrm{melanomas}(0.00-1.00 \mathrm{~mm}$ thickness $)$ is $92 \%$ but only $50 \%$ in patients with T4 melanomas ( $>4.00 \mathrm{~mm}$ thickness). Primary tumor ulceration was only added to the staging criteria in 2009 - on the basis of the observation that survival rates with an ulcerated melanomas are consistently lower than for nonulcerated melanoma of equivalent $\mathrm{T}$ category; for each thickness, the outcome for an ulcerated melanoma proved to be similar to that of patients with a non-ulcerated melanoma of the next higher thickness (T) category in the 2009 staging manual. Increasing mitotic rate (defined as the number of mitoses per square millimeter) marks a more aggressive lesion and is associated with worse survival at every $\mathrm{T}$ category. In fact, registry data suggest that mitotic rate is the second most powerful predictor of survival, with a potential for negatively impacting survival even in otherwise favorable prognosis disease states. Considering nonulcerated T1 melanomas, 10-year survival rates are $95 \%$ for lesions with mitotic rate of $<1$ / $\mathrm{mm}^{2}$ and drop to $88 \%$ for lesions with mitotic rate of $\geq 1 / \mathrm{mm}^{2}$. Mitotic rate has also replaced Clark's depth of invasion in defining T1 lesions - T1a melanoma refer to $\unlhd .00$ $\mathrm{mm}$ thick non-ulcerated lesions with mitotic rate $\leq 1 / \mathrm{mm}^{2}$ with $\mathrm{T} 1 \mathrm{~b}$ lesions referring to $\unlhd 1.00 \mathrm{~mm}$ thick melanomas that are either ulcerated or have a mitotic rate $>1 / \mathrm{mm}^{2}$. 
Melanoma has a predilection for lymphatic seeding and the risk of regional lymph node involvement increases with tumor thickness - 2-5\% for Breslow's depth $\leq 1.00 \mathrm{~mm}$ but $34 \%$ for T4 lesions [12]. Increasing lymph node tumor burden is associated with worse survival the 5-year survival of stage III patients, sub-divided by the extent of lymph node involvement shows a steady decline from $78 \%$, to $59 \%$, and $40 \%$ for stages IIIA, IIIB, and IIIC respectively. In the 7th edition AJCC staging system, nodal micro-metastases can be defined by immunohistochemical staining rather than by $\mathrm{H} \& \mathrm{E}$ alone and the concept of a minimum threshold of lymphatic tumor burden was abolished with any degree of involvement considered significant—whether micro- or macro- metastatic.

When considering systemic metastatic disease, the number of metastatic sites, the sites of distant metastases and the serum lactate dehydrogenase (LDH) enzyme level are important prognostic factors. LDH expression may be related to the progression of melanomas - being barely detectable in nevi but strongly expressed in thick primary melanoma and in metastatic melanoma [13]. The significant drop-off in 1-year survival between patients with M1a and M1b disease (62\% and 53\%) compared to M1c disease (33\%) underscores the importance of detecting non-lung visceral metastases and assaying LDH in prognostication.

\section{Indications for Adjuvant Therapy}

By definition, adjuvant therapy is offered after definitive surgical treatment has removed all detectable disease and is given with the intent of reducing relapse risk due to occult disease.

Currently, adjuvant therapy with the FDA-approved regimen of high-dose interferon (HDI) for one year or enrollment in a clinical trial should be considered for patients whose estimated risk of recurrence exceeds $30 \%$; i.e. patients with node-positive melanoma and T3b or T4 node-negative disease.

\section{Immunotherapy}

Cancer immunotherapy has evolved considerably since Coley's observations of tumor shrinkage with inoculation of bacterial products into tumors. For melanoma, the lack of effective chemotherapeutic options, and the observations of antitumor response rates of $15 \%$ to $20 \%$ with systemic administration of IFN-a led to the consideration of adjuvant application of this agent. The biology of this agent and the data that led to the FDA-approval of IFN-a for adjuvant treatment of stage IIB-III resectable melanoma in 1995 follows.

\section{Immunotherapy: IFN - Mechanism of Action}

Based on functional and structural differences, IFNs are sub-classified into types I and II. Type 2 IFN (IFN- $\gamma$ ) is produced by $\mathrm{T}_{\mathrm{H} 1}$ cells and exerts a weak anti-viral and anti-tumor effect and up-regulates $\mathrm{T}_{\mathrm{H} 2}$ activity. Type 1 IFNs (IFN- $\alpha$, IFN- $\beta$ and IFN- $\omega$ ) are produced primarily by dendritic cells in response to infectious agents. Signaling via the IFN-a receptor, type 1 IFNs connect the adaptive and innate arms of the immune response and have potent apoptotic, anti-proliferative, anti-angiogenic and immunoregulatory properties.

IFN-a's mechanism of action in melanoma is thought to be immunomodulatory rather than directly cytotoxic or anti-angiogenic. Accumulated evidence suggests that anti-tumor immunity is abrogated by the tumor through immunosuppressive circuits including the cascade triggered by constitutive activation of STAT3 in the tumor [14] with the elaboration of VEGF, IL-10 and TGF $\beta$, as well as regulatory T (Treg) cells within the tumor microenvironment [15]. Additionally, it is known that type 1 IFNs play a critical role in Tcell priming, especially that mediated by dendritic cells, an effect which is subsequently diminished as tumor outgrowth occurs and may be reversed by IFN administration supported by observations from Gajewski and colleagues who have reported that the in vivo 
expression of IFN- $\alpha$ or IFN- $\beta$ by retroviral transduction can lead to total rejection of melanoma in a murine model [16]. Observational data from a clinical trial involving patients with stage IIIB/C melanoma given neo-adjuvant high-dose interferon (HDI) prior to definitive lymph node dissection suggested that IFN-a caused an influx of Tcells and dendritic cells to a degree that correlated directly with responses [17].

\section{Immunotherapy: IFN - Clinical Development}

Following initial evidence of activity of IFN-a in metastatic melanoma, multiple phase II trials were conducted and response rates observed were similar to single-agent chemotherapy ( 13-24\%). Notably, durable responses that lasted years led to investigations in the adjuvant setting for high-risk resected melanoma. The studies that followed have differed primarily in relation to dosage of IFN-a (low-dose $\leq 3 \mathrm{MU} / \mathrm{dose}$, intermediate-dose 5-10 MU/dose, and high-dose $\geq 10 \mathrm{MU} / \mathrm{dose}$ ) as well as the sub-species of IFN-a used [IFN$a 2 a$, IFN-a $2 b$ and IFN-a2c) and the schedule employed for therapy; these are summarized in Table I.

The initial US Cooperative Group trials primarily involved HDI and of these, two in particular had significant therapeutic results in relation to reduction in recurrence and/or mortality. The North Central Cancer Treatment Group (NCCTG) trial tested a regimen of $20 \mathrm{MU} / \mathrm{m} 2$ dose of IFN-a 2a administered intra-muscularly thrice weekly for twelve weeks for stage II and III disease while the Eastern Cooperative Group (ECOG) trial (E1684) tested an induction phase of one month of daily intravenous (IV) IFN-a $2 b$ at $20 \mathrm{MU} / \mathrm{m}^{2}$, followed by 11 months of maintenance therapy at $10 \mathrm{MU} / \mathrm{m}^{2}$ dosage sub-cutaneously (SC) 3 days a week [18-19]. The NCCTG trial demonstrated improvements in median disease free survival (DFS) and OS that were non-significant, with higher risk patients appearing to benefit disproportionately. E1684 was a landmark study - demonstrating statistically significant improvements in both DFS and OS among patients with high-risk disease (T4 primaries or regional lymph node metastases) in a randomized controlled setting that paved the way for FDA approval of HDI in high-risk T4 (stage IIB) and N1-2 (IIIA/B) patients as defined by this trial.

Balanced against these benefits, the toxicity observed with $67 \%$ incidence of Grade III toxicity, $9 \%$ incidence of Grade IV toxicity, and 2 early treatment-related hepatotoxic deaths in E1684 raised concerns over the tolerability and compliance with this regimen and prompted investigators to study lower doses of IFN-a. A variety of alternative regimens utilizing lower doses of IFN-a were then tested including the very low dose regimen (1 MU SC every other day) tested in EORTC 18871 [20] (stage IIB/III) and the low dose regimen (3 MU SC thrice weekly) tested in WHO Melanoma Program Trial 16 [21] (stage III), Scottish Melanoma Cooperative Group trial [22] (stage IIB/III), UKCCCR AIM-High trial [23] (stage IIB/III), E1690 [24] (T4, N1) and the 2010 German DeCOG study [25] (T3anyN) - without any overall survival benefit being observed. The 2008 German DeCOG study [26] demonstrated a survival benefit for LDI but was powered primarily to assess the benefit of combination LDI with dacarbazine and not designed to evaluate the low dose regimen per se.

Two trials of the low dose regimen by Austrian (AMCG trial [27]) and French (FCGM trial [28]) groups were carried out in patients with stage II disease (T2-4NOM0) - while the Austrian trial reported improvements in DFS but not OS, the French trial reported significant prolongation of DFS and OS. However, given the overall good prognosis of stage II disease, the relative cost-benefit ratio of this strategy is questionable. A number of trials have tested intermediate dose IFN-a and EORTC 18952 [29] (stage IIB/III) demonstrated a $7.2 \%$ increase in DMFS, but this was not statistically significant and no OS benefit was observed. 
An interesting alternative approach to adjuvant therapy was taken by the Italian Melanoma Intergroup in a randomized phase III study that enrolled 336 patients with stage III disease to received either standard HDI or intensified HDI (IHDI - IFN-a $2 b 20 \mathrm{MU} / \mathrm{m}^{2}$ intravenously 5 days a week for 4 weeks every other month for 4 cycles) [30]. At the 5 year mark, there were no statistically significant differences in either RFS or OS and similar toxicity rates amongst patients in both groups. Although mature survival data has yet to emerge from evaluation of intensive IHDI this regimen may be more tolerable than conventional HDI.

Survival analysis in E1684 suggested that the greatest reduction in relapse occurred relatively early arguing for the importance of the regimen's induction phase - and was sustained thereafter to more than a decade. The benefit of an abbreviated course of one month of therapy has been prospectively tested in the Hellenic study (induction HDI only versus induction and maintenance) and in E1697 (4 weeks of HDI versus observation) [31-32]. The Hellenic trial tested non-inferiority of an induction-only arm and suggested little difference between this and the longer one-year treatment with the modified dosage of IFN tested, on the basis that the 3-year relapse rate in the induction only arm was less than $15 \%$ higher than the relapse rate of the one-year group. However the relatively small size, use of a reduced dose IFN- $a$ regimen, and the lack of an observation arm make these results hard to interpret. At the interim analysis of E1697 after 1150 of a planned 1420 patients were randomized, the study was closed for futility. Taking these results together, it is unlikely that durable benefits are associated with one month of IV induction therapy alone, and therapy with the full year as in E1684 is now recommended.

Given the accumulated evidence for adjuvant HDI, there is abundant evidence that HDI results in an approximate $30 \%$ reduction in RFS with a reduction in mortality that is smaller in two studies (E1684 and E1694). Retrospective data from meta-analyses [8], systematic reviews $[7,10]$ and a pooled data analysis [33] consistently support the conclusion that HDI increases DFS with a smaller benefit upon OS.

\section{Immunotherapy: IFN - Pegylated IFN}

Pegylated IFN- $a$ was first utilized in the treatment of hepatitis B/C and pharmacokinetic data from hepatitis studies and phase I/II trials in oncology supported the ability to maintain therapeutic dose levels with once weekly subcutaneous injections [34]. Trials conducted by the EORTC have demonstrated RFS benefits in the adjuvant setting that has led to the regulatory approval of this agent for adjuvant treatment of stage III melanoma in the US.

EORTC 18991 investigated the use of pegylated IFN-a2b in patients with resected AJCC stage III melanoma in a randomized phase III trial [35]. Therapy comprising 'induction' doses that are somewhat higher (Peg-IFN-a2b SC $6 \mu \mathrm{g} / \mathrm{kg}$ a week for 8 weeks) followed by maintenance doses (weekly SC injections at $3 \mu \mathrm{g} / \mathrm{kg}$ for 5 years) was compared to observation. Recently presented 7.6 year follow-up data showed an improved RFS in the treatment arm with no difference in OS or distant metastases-free survival (DMFS). Subgroup analysis has revealed that patients with microscopic nodal metastases and ulcerated primaries appeared to benefit disproportionately in terms of RFS/OS and DMFS. This subset analysis finding was buttressed by pooled analysis of EORTC 18952/18991 that spurred interest in investigating adjuvant therapy with Peg IFNa in this sub-group of patients [36]. EORTC 18081 is a prospective trial that will randomize patients with ulcerated primaries to either pegylated IFN- $a 2 b$ versus observation. For the present, the use of pegylated IFN- $a 2 b$ in microscopic nodal disease (AJCC IIIA) may be considered as an alternative to HDI for patients unwilling to consider HDI. 
Low dose pegylated IFN-a2b was compared to LDI in a prospective phase III European Association of Dermato-Oncology (EADO trial) that enrolled patients with resected stage IIA-IIIB melanoma ( $\mathrm{T} \geq 1.5 \mathrm{~mm}$, without clinically detectable nodal disease). Patients were randomized to either LDI (3MU SC thrice weekly) for 18 months or low dose pegylated IFN-a 2b (100mcg SC once weekly) for 36 months. No differences between groups in terms of RFS, OS or DMFS were found, although results were likely affected by the $72 \%$ dropout rate secondary to serious adverse events in the pegylated IFN-a $2 \mathrm{~b}$ arm $(44.6 \%$ versus $26.6 \%)[37]$.

\section{Immunotherapy: IFN - The Future}

The accumulated evidence suggests that adjuvant IFN-a2b therapy has a remarkably sustained impact on RFS with a lesser impact on OS, the basis of which has never been adequately examined in terms of the actual basis of late mortality beyond 10 years. Various groups have attempted to identify prognostic markers of interferon benefit to focus this therapy upon patients most likely to benefit.

Recent European data suggests that patients with certain clinical features (ulcerated nodepositive disease) benefit disproportionately from IFN- $a 2 b$ therapy although multiple prior ECOG and US Intergroup trials have not similarly identified ulceration as a predictor of benefit for higher dosages of IFN. This is planned for prospective evaluation in EORTC 18081, and results of EORTC 18081 are awaited to address this question.

Since the most mature data for high-dose IFN do not suggest any difference in relapse risk reduction amongst patients with AJCC stage IIB, IIIA, and IIIB resectable disease, it has been reasonable to impute benefit for high-dose IFN in stage IV patients from these results. Thus, if the risk reduction for a patient with stage IIIA disease and stage IIIB disease is $\sim 33 \%$, the benefit of this therapy for resectable stage IV may be similar. Conversely, the benefit of adjuvant therapy may diminish with more advanced disease, as has been reported with intermediate dosage regimens of IFNa2b and Peg IFN. The EORTC 18952 intermediate dose IFN-a2b trial and EORTC 18991 Peg-IFN trial demonstrated activity chiefly in stage IIIA (N1) patients, and negligible benefits amongst stage IIIB (N2) patients with notable differences in the patterns of improvement observed [29,38]. Although both trials recruited high risk patients, the Nordic IFN trial enrolled more node-positive patients (79\%-81\%) compared to EORTC 18952 (74\%-75\%). EORTC 18952 demonstrated that the benefits of PEG-IFN were greater in stage IIB/C patients compared to node-positive patients implying that low preoperative tumour burden was predictive of IFN response. However, the Nordic IFN trial suggested that patients with node-positive disease benefited more than node-negative patients, especially when treated with a longer duration of therapy. Assuming that IFN does not exert differential effects across stages, one would assume that any benefit would be more obvious in higher risk patients who have a higher rate of adverse events and a worse prognosis.

There has been a paucity of trials that have addressed the specific issue of adjuvant therapy in the highest risk category - resected stage IIIB-C/IV disease. The failed phase III study of Canvaxin ${ }^{\circledR}$ in resected stage IV melanoma patients [39] suggests that Canvaxin had no benefit as adjuvant therapy of either resectable stage III or stage IV disease. The role of adjuvant immunotherapy with high-dose IFN has been evaluated in the highest risk category of resected stage IIIB and IV melanoma in one phase II trial E2696 (adjuvant HDI combined with GM2 vaccination compared to GM2 alone [40]. However, the phase III E4697 (GMCSF versus placebo) and a retrospective case-control study from Mayo that studied the use of adjuvant GM-CSF therapy following surgical resection have suggested that there may be an opportunity to benefit patients with resectable stage IV disease with the use of GM-CSF adjuvant immunotherapy [41-42]. Specifically in E4697, although the study overall 
indicated no benefit for GM-CSF over placebo, when stratified by stage, the investigators observed that patients with stage IV M1a/b disease had a significant trend towards improvement in both DFS and OS whereas stage III patients -had no apparent difference. The hazard ratio for RFS benefit of GM-CSF over placebo in Stage IV resected patients was 0.74 while it was 0.92 for Stage III while HR for survival benefit in Stage IV patients was 0.72 and for stage III it was 0.97. This result in patients with Stage IV and the highest level of risk should be evaluated further in the future. E1609, which is selectively accruing high risk patients with resected stage IIIB/IV disease and is prospectively randomizing them to adjuvant therapy with either ipilimumab or HDI, will hopefully provide some clarification in this area.

Autoimmune manifestations following interferon therapy have been associated with improved prognosis in both the E2696 and E1694 US intergroup trials [43-44]. These were prospectively validated by the results of Gogas et al, where the authors showed that patients receiving IFN-a for either the full year or an abbreviated schedule of 1 month were more likely to develop autoimmune clinical manifestations or auto-antibodies and that patients who developed autoimmune manifestations had improved DFS, OS and reduced rates of relapse and mortality compared to those who did not do so [45]. Other studies using different methods have shown conflicting results and current studies aim to define the immunogenetic basis of autoimmune events [46].

Multiple other candidate biomarkers of interferon response have been studied, including methylthioadenosine phosphorylase (MTAP) expression, YKL-40, S100B, melanomainhibiting activity (MIA) and tumor-associated antigen 90 immune complex (TA90IC). Notably prospective data is lacking for these biomarkers and prospective validation would be important to obtain. This is reviewed elsewhere in detail [47].

Approximately 40 to $60 \%$ of advanced melanomas possess activating mutations in BRAF, and BRAF inhibitors induce dramatic antitumor responses in these patients. Recently presented data suggests that BRAF mutations contribute to immune escape and that BRAF inhibition increases expression of melanocyte differentiation antigens (MDA) and improved recognition by antigen-specific T-cells [48]. Given the known effects of interferon on T-cell function, combining interferon with BRAF inhibition may be synergistic on multiple grounds. Other interferon combinations under investigation include HDI and KW2871, a monoclonal antibody targeting GD3 which is a ganglioside expressed on the surface in over 95\% of melanomas. A phase II trial of the latter combination is presently in active accrual (NCT00679289). Although such trials initially only involve patients with metastatic disease, should efficacy be established, it is not unreasonable to consider extending this to the adjuvant setting.

\section{Immunotherapy: Vaccine Therapy}

The goal of vaccine therapy is to elicit durable anti-tumor effects that result in sustained clinical responses in a significant proportion of patients treated - an approach first pursued in melanoma by Morton in 1967.

Melanoma vaccines are divided based on the type of the antigen(s) incorporated - peptide, ganglioside and whole cell/cell lysate. Peptide vaccines have utilized melanocyte lineage antigens (such as MART-1/Melan-A, gp100 and tyrosinase) that are recognized by cytotoxic T lymphocytes in conjunction with HLA-A2.1 and elicit a direct cytotoxic T-cell response.

Vaccines based on peptide antigens have been studied in large multicenter ECOG trials that have generally recruited pre-treated patients with advanced metastatic melanoma. Although only a few patients demonstrated immune responses to any of the peptides, those who did so 
tended to develop increased T-cell production of IFN- $\gamma$ and had survival times that were far greater than that of patients who did not develop immunity to peptide epitopes.

Of the seven large randomized trials of adjuvant allogeneic melanoma cell-based vaccines that have been conducted to date, most have been disappointingly negative. In the United States, the trial of the Melacine vaccine in stage II patients and the two trials of the Canvaxin vaccine in stage III and resected stage IV patients were negative and the latter showed a trend toward adverse impact. In Europe, the EORTC initially published data suggesting prolonged DFS in patients treated post-operatively with GM2/BCG vaccination [49]. However, when investigated prospectively in EORTC 18961, a trend toward adverse DMFS and OS outcomes led to trial termination at $2^{\text {nd }}$ interim analysis for safety concerns [50]. More mature data has suggested no significant difference in any outcome in this trial. An Australian study did demonstrate a non-significant increase in OS and RFS among patients treated with a vaccinia melanoma cell lysate vaccine following definitive surgery, although this has not been reproduced by others [51].

The MAGE-A3 antigen is expressed in a wide variety of malignancies including melanoma but is not detected in most normal tissues except for testis and placenta [52]. Unlike peptides that demonstrate MHC-restricted activity, MAGE-A3 protein vaccination is suitable for a majority (70\%) of melanoma patients whose tumors express this antigen and elicits a broad range of non MHC-restricted T-cell responses. Following a phase I/II study that demonstrated MAGE-3-specific antibody and T-cell responses following vaccination in patients with MAGE-3-positive tumors [53], a randomized phase III study known as DERMA has completed enrollment of patients with stage III nodal metastases and detectable MAGE-3- expression in the resected lymph nodes. Results from this trial are pending.

Morton and colleagues developed a polyvalent cultured melanoma cell vaccine (Canvaxin $\left.{ }^{\circ}\right)$ that was evaluated in stage III melanoma patients following complete resection in a retrospective study that suggested an improvement in median and 5-year specific OS for vaccinated patients [54]. However, when Canvaxin ${ }^{\circledR}$ was compared to BCG alone in a phase III RCT for patients with resected stage III/IV melanoma, Canvaxin ${ }^{\circledR}$ vaccination failed to improve DFS and OS. In fact, the survival for vaccinated patients was diminished, possibly secondary to vaccine induced immunosuppression that led to early closure of the trial by the DSMB for futility [55].

\section{Immunotherapy: CTLA-4 Blockade and Immunomodulatory Agents}

Two CTLA-4 blocking monoclonal antibodies have been evaluated in the clinic ipilimumab and tremelimumab. Tremelimumab (Pfizer/MedImmune) is a fully humanized non-complement fixing $\operatorname{IgG} 2$ monoclonal antibody that was initially evaluated in a broad variety of malignancies though subsequent phase II trials focused on melanoma after promising results in phase I studies. In the phase II trial of patients with advanced relapsed or refractory melanoma who received tremelimumab dosed at $15 \mathrm{mg} / \mathrm{kg}$ (IV every 90 days), a $6.6 \%$ objective response rate (ORR) was observed, all of which were durable and lasted more than 6 months [56]. However, the initial optimism generated by the phase II results were followed by negative results in the registration phase III trial against chemotherapy (temozolamide or dacarbazine) in which tremelimumab did not confer a significant survival advantage at interim analysis (OS tremelimumab 11.76 months versus chemotherapy 10.71 months) and led to early closure [57]. It is also possible that the results of this trial were affected by the open label nature of the study, and its restriction of enrollment to patients with LDH levels less than twice the upper limit of normal. Crossover of patients who were assigned to chemotherapy but then pursued anti-CTLA4 therapy in expanded-access 
ipilimumab trials may also have reduced the apparent treatment benefit and diluted the OS statistic.

Ipilimumab (Medarex Inc/Bristol-Myers Squibb) is a fully humanized IgG1 $\kappa$ monoclonal antibody that blocks CTLA-4. Two recently published phase III studies evaluated the use of ipilimumab in patients with metastatic melanoma in different settings (first and second line) against different comparators (gp100 vaccine and dacarbazine) and arrived at similar conclusions. This data is tabulated in Table II.

The earlier phase III MDX010-20 trial compared ipilimumab alone (at a dose of $3 \mathrm{mg} / \mathrm{kg}$ ), ipilimumab plus a peptide vaccine, and vaccine plus placebo; this trial demonstrated a significant increase in survival rates at both 12 (46\% versus $25 \%)$ and 24 months (24\% versus $14 \%$ ) compared to the vaccine comparator [58]. The dose of $3 \mathrm{mg} / \mathrm{kg}$ was based on results of the randomized phase II dose-ranging study that demonstrated tolerability of the $10 \mathrm{mg} / \mathrm{kg}$ dose level [59]. More recently, the results of the second randomized phase III CA184-024 trial that compared ipilimumab (at a dose of $10 \mathrm{mg} / \mathrm{kg}$ ) plus dacarbazine $(850$ $\mathrm{mg} / \mathrm{m}^{2}$ ) to dacarbazine with placebo were released at ASCO 2011 [60]. This trial enrolled chemotherapy-naïve patients and a similar schedule MDX010-20 involving 4 induction doses followed by monthly maintenance for responders was utilized. The ipilimumab/ dacarbazine combination was associated with a survival advantage that was sustained at three years [61].

With the success of ipilimumab in the metastatic setting, trials investigating the potential for ipilimumab in the adjuvant setting have been undertaken. These include the ECOG sponsored intergroup trial E1609 based in the United States and EORTC 18071 conducted in Europe comparing ipilimumab against the reference HDI in the US and against placebo in Europe. Accrual for EORTC 18071 is complete and results are pending while for E1609 accrual is now active, but the trial results are not anticipated until 2014 or after.

Given the high cost and toxicity of these therapies, clinically relevant biomarkers or predictors of response would be invaluable to guide therapeutic decision making. Unpublished MDX010-20 study data suggests that absolute lymphocyte counts (ALC) appear to increase in a dose-dependent fashion with ipilimumab therapy with high baseline ALC values being associated with improved outcomes. Hamid et al have reported that elevated tumor infiltrating lymphocyte (TIL), Treg and indoleamine 2,3-dioxygenase (IDO) levels in pre-treatment biopsy specimens are correlated with improve outcomes in patients with metastatic disease receiving ipilimumab therapy [62].

\section{Immunotherapy: Other Emerging Checkpoints}

Other potential immunomodulatory targets include anti-PD-1, anti-OX44 and anti-4-1BB antibodies. Along with CTLA4, the programmed death-1 (PD-1) receptor is a negative costimulatory circuit that down-regulates T-cell activation and response. PD-1 ligand (PD-L1) is expressed on the surface of melanoma cells, B-cells, dendritic cells and macrophages and PD-L1 upregulation appears to depend on toll like receptor 4 (TLR-4) based signaling as well as being induced by IFNa. Increased PD-1 interaction has been implicated in maintaining immune tolerance through diminished T-cell effector function and PD-1 blockade has been shown to promote the generation of melanoma antigen-specific cytotoxic T-cells (CTL) and overcome Treg mediated suppression [63]. OX44 and CD137 are T-cell targets that up-regulate the immune response. Agonistic antibodies anti-OX44 and anti-4-1BB may increase the T-cell response against melanoma. 
Thus far, the use of these agents has been limited to early phase trials in metastatic disease and formal results of phase III trials are awaited prior to the extension of these agents into the adjuvant setting.

\section{Chemotherapy}

Several trials have assessed the use of adjuvant chemotherapy following surgical resection in high-risk patients. Multiple small non-randomized single-center studies have assessed various options including megestrol acetate, vitamin A, vindesine and dacarbazine - both singly, and in combination with BCG. However, despite suggestions of benefit, none has been demonstrated in subsequent randomized controlled trial. These data are summarized in Table III and is reviewed in detail elsewhere [64].

Two trials of chemotherapeutic combinations have demonstrated increased RFS (but not OS) while no benefit was observed with the combination of DTIC/BCG in E1673 [65]. The 2008 phase III DeCOG study compared a combination LDI and dacarbazine to adjuvant LDI alone following completion lymph node dissection [66]. Although the LDI only arm demonstrated a survival benefit, this trial was not powered adequately to assess the benefit of low dose IFN over observation.

Although the combination of biologics with chemotherapy (biochemotherapy) has been associated with improvements in response rate and PFS in the metastatic setting, no OS benefit has been observed compared to dacarbazine monotherapy. S0008 is an intergroup phase III study organized by the Southwest Oncology Group (SWOG) testing the benefit of biochemotherapy vs. HDI. The study enrolled resectable stage IIIB and IV patients and randomized subjects to either 1 year of HDI or 3 cycles of cisplatin, vinblastine, DTIC, IL-2 and interferon given monthly. As both the IL-2 and interferon were dosed substantially below their individual maximally tolerated doses, this trial is really an evaluation of the effect of chemotherapy modulated by IFN/IL-2 - although completed, trial results are still pending and expected in 2012.

\section{Radiotherapy}

In melanoma, RT is rarely indicated in the primary setting as surgical excision with wide margins provides valuable diagnostic and prognostic information. However, the risk of local relapse despite CLND is $15-20 \%$ and elevated to $30-50 \%$ for patients with extra-capsular lymph node extension (ECE), involvement of 4 or more nodes, or bulky disease (exceeding $3 \mathrm{~cm}$ in size), cervical lymph node location, and recurrent disease. In such patients, adjuvant RT may be valuable especially if patients are intolerant of HDI therapy.

Multiple non-randomized trials have evaluated the use of RT in this setting and have generally concluded that adjuvant RT was associated with improved local and regional control rates without any survival benefit. Similar to the data from breast and prostate cancer, hypofractionation appears to be equally efficacious as standard dosing in treating melanoma [67].

ANZMTG 01.02/TROG 02.01 is a more recent prospective multi-center phase III trial involving clinical sites in Australia, New Zealand and the Netherlands [68]. 217 patients from 16 centers were randomized to observation versus regional nodal basin RT (48Gy in 20 fractions). Although RT use resulted in a statistically significant improvement in regional control (HR 1.77, 95\% CI 1.02-3.08, p=0.041), it had no impact on survival. In fact, RT use was actually associated with poorer survival ( 31 months vs. 47 months, $\mathrm{p}=0.14$ ). There are no insights to this paradoxical observation at this time. Several follow up studies including RTOG 9302 (phase III study of post-operative adjuvant RT in patients with cutaneous melanomas of head and neck) were planned but have since been stopped secondary to non- 
accrual. There remains a paucity of prospective data evaluating the use of RT especially in patients with advanced regional nodal disease and extra-capsular disease extension in whom the role of HDI in preventing systemic or locoregional recurrence is not well defined.

\section{Future questions/Conclusion}

Results of multiple US and European intergroup studies (E1684, E1690, E1694, EORTC 18952 and EORTC 18991) have shown that adjuvant IFN-a improves RFS though the impact on survival appears to be less overall, and for HDI where two trials show an impact on OS, the benefit diminishes after 10 years. For pegylated IFN- $\alpha$ the impact has been solely upon RFS. Identifying factors that may predict responsiveness of patients to this modality is vital to improve the therapeutic index. Thus far, we know that certain features of the primary tumor (ulcerated primaries and/or microscopic node positive disease) may predict the benefit of lower dosage regimens and the development of auto-immune manifestations during therapy are associated with therapeutic response. The former is being prospectively validated in an EORTC trial (18081).

Despite the high incidence of grade 3/4 adverse events observed in the phase III trials of ipilimumab in patients with metastatic melanoma, the presence of durable complete responses and significant improvements in RFS and OS have prompted adjuvant studies by EORTC and the US intergroup.

Here, as with IFN-a, biomarkers of response and treatment effect are needed. In the metastatic setting at least, elevated TILs, Treg and IDO levels in pre-treatment biopsies have been suggested to predict response to ipilimumab. Two separate groups of investigators have confirmed that ipilimumab therapy results in greater frequencies of circulating Tregs and increased expression of proliferation and polarization markers in CD4 ${ }^{+}$and $\mathrm{CD} 8^{+} \mathrm{T}$-cells which may serve as indicators of the pharmacodynamic effects of ipilimumab [69]. The roles of CRP and of MDSC have been supported as biomarkers of CTLA-4 in the treatment of adjuvant disease and require evaluation in the adjuvant setting [70-71].

A different paradigm may been seen in the molecularly targeted therapy of melanoma with BRAF, and MEK inhibitors, where high response rates and PFS and OS benefits have been observed in metastatic melanoma prompting consideration in the adjuvant setting. This is especially interesting since BRAF inhibition results in increased immune recognition and may abrogate tumor-mediated immune tolerance, allowing for rational combinations with a variety of immunotherapeutics. However, limiting adverse events such as rash, arthralgias and cutaneous squamous cell carcinomas, the rapid development of BRAF resistance within months of initiating treatment, and the lack of data to demonstrate the kinetics of the immune alterations thus far pose obstacles to this approach. The use of neo-adjuvant study designs in which patients would have tumor tissue biopsy before and after a limited exposure to BRAFi therapy will be crucial to understand the potential range of effects that this agent may have, of relevance to the adjuvant therapeutic arena. These trial designs are now being developed, and may draw from the experience with imatinib in GIST (72-73).

Multiple scientific advances have been exploited in the clinic with dramatic results. However, the rational basis combinations and sequence of these agents to achieve significant and durable benefits in overall and relapse-free survival remain a focus of intense investigation. For now, the management of patients with high-risk melanoma remains highdose IFN- $a$ or enrollment into a clinical trial. 


\section{Acknowledgments}

The project described above was supported by Award Number P50 CA121973 from the National Cancer Institute. The content is solely the responsibility of the authors and does not necessarily represent the official views of the National Cancer Institute or the National Institutes of Health.

\section{References}

1. Howlader, N.; Noone, AM.; Krapcho, M., et al. [29 November 2011] SEER Stat Fact Sheets: Melanoma of the Skin. National Cancer Institute SEER Database. http://seer.cancer.gov/statfacts/ $\mathrm{html} / \mathrm{melan} \cdot \mathrm{html}$

2. Hodi FS, O’Day SJ, McDermott DF, et al. Improved survival with ipilimumab in patients with metastatic melanoma. N Engl J Med. 2010 Aug 19; 363(8):711-23. [PubMed: 20525992]

3. Robert C, Thomas L, Bondarenko I, et al. Ipilimumab plus dacarbazine for previously untreated metastatic melanoma. N Engl J Med. 2011 Jun 30; 364(26):2517-26. [PubMed: 21639810]

4. Chapman PB, Hauschild A, Robert C, et al. for BRIM-3 Study Group. Improved survival with vemurafenib in melanoma with BRAF V600E mutation. N Engl J Med. 2011 Jun 30; 364(26):250716. [PubMed: 21639808]

5. Garbe C, Eigentler TK. Diagnosis and treatment of cutaneous melanoma: state of the art 2006. Melanoma Res. 2007; 17:117-127. [PubMed: 17496787]

6. Tarhini AA, Kirkwood JM. Clinical and immunologic basis of interferon therapy in melanoma. Ann N Y Acad Sci. 2009 Dec.1182:47-57. [PubMed: 20074274]

7. Lens MB, Dawes M. Interferon alfa therapy for malignant melanoma: a systematic review of randomized controlled trials. J Clin Oncol. 2002; 20:1818-1825. [PubMed: 11919239]

8. Wheatley K, Ives N, Hancock B, et al. Does adjuvant interferon-alpha for high-risk melanoma provide a worthwhile benefit? A meta-analysis of the randomised trials. Cancer Treat Rev. 2003; 29:241-252. [PubMed: 12927565]

9. Wheatley K, Hancock B, Gore M, et al. for International Malignant Melanoma Collaborative Group. Interferon-\{alpha\} as adjuvant therapy for melanoma: an individual patient data meta-analysis of randomised trials. J Clin Oncol. 2007; 25(18 suppl) Abstract 8526.

10. Mocellin S, Pasquali S, Rossi CR, et al. Interferon alpha adjuvant therapy in patients with high-risk melanoma: a systematic review and metaanalysis. J Natl Cancer Inst. 2010; 102:493-501. [PubMed: 20179267]

11. Balch CM, Buzaid AC, Soong SJ, et al. Final version of the American Joint Committee on Cancer staging system for cutaneous melanoma. J Clin Oncol. 2001; 19(16):3635-3648. [PubMed: 11504745]

12. Kettlewell S, Moyes C, Bray C, et al. Value of sentinel node status as a prognostic factor in melanoma: prospective observational study. BMJ. 2006 Jun 17.332(7555):1423. [PubMed: 16735303]

13. Zhuang L, Scolyer RA, Murali R, et al. Lactate dehydrogenase 5 expression in melanoma increases with disease progression and is associated with expression of Bcl-XL and Mcl-1, but not Bcl-2 proteins. Mod Pathol. 2010 Jan; 23(1):45-53. [PubMed: 19838163]

14. Kirkwood JM, Farkas DL, Chakraborty A, et al. Systemic interferon-alpha (IFN-alpha) treatment leads to Stat3 inactivation in melanoma precursor lesions. Mol Med. 1999 Jan; 5(1):11-20. [PubMed: 10072444]

15. Gajewski TF. The expanding universe of regulatory T cell subsets in cancer. Immunity. 2007 Aug; 27(2):185-7. [PubMed: 17723212]

16. Gajewski TF, Fuertes M, Spaapen R, et al. Molecular profiling to identify relevant immune resistance mechanisms in the tumor microenvironment. Curr Opin Immunol. 2011 Apr; 23(2): 286-92. [PubMed: 21185705]

17. Moschos SJ, Edington HD, Land SR, et al. Neoadjuvant treatment of regional stage IIIB melanoma with high-dose interferon alfa-2b induces objective tumor regression in association with modulation of tumor infiltrating host cellular immune responses. J Clin Oncol. 2006 Jul 1; 24(19): 3164-71. [PubMed: 16809739] 
18. Creagan ET, Dalton RJ, Ahmann DL, et al. Randomized, surgical adjuvant clinical trial of recombinant interferon alfa-2a in selected patients with malignant melanoma. J Clin Oncol. 1995 Nov; 13(11):2776-83. [PubMed: 7595738]

19. Kirkwood JM, Strawderman MH, Ernstoff MS, et al. Interferon alfa- $2 b$ adjuvant therapy of highrisk resected cutaneous melanoma: the Eastern Cooperative Oncology Group Trial EST 1684. J Clin Oncol. 1996; 14:7-17. [PubMed: 8558223]

20. Kleeberg UR, Suciu S, Bröcker EB, et al. for EORTC Melanoma Group in cooperation with the German Cancer Society (DKG). Final results of the EORTC 18871/DKG 80-1 randomised phase III trial. rIFN-alpha2b versus rIFN-gamma versus ISCADOR $M$ versus observation after surgery in melanoma patients with either high-risk primary (thickness $>3 \mathrm{~mm}$ ) or regional lymph node metastasis. Eur J Cancer. 2004 Feb; 40(3):390-402. [PubMed: 14746858]

21. Cascinelli N, Belli F, MacKie RM, et al. Effect of long-term adjuvant therapy with interferon alpha-2a in patients with regional node metastases from cutaneous melanoma: a randomised trial. Lancet. 2001; 358(9285):866-869. [PubMed: 11567700]

22. Cameron DA, Cornbleet MC, Mackie RM, et al. Adjuvant interferon alpha $2 b$ in high risk melanoma-the Scottish study. Br J Cancer. 2001; 84(9):1146-1149. [PubMed: 11379605]

23. Hancock BW, Wheatley K, Harris S, et al. Adjuvant interferon in high-risk melanoma: the AIM HIGH Study-United Kingdom Coordinating Committee on Cancer Research randomized study of adjuvant low-dose extended-duration interferon alfa-2a in high-risk resected malignant melanoma. J Clin Oncol. 2004; 22(1):53-61. [PubMed: 14665609]

24. Kirkwood JM, Ibrahim JG, Sondak VK, et al. High- and low-dose interferon alfa-2b in high-risk melanoma: first analysis of intergroup trial E1690/S9111/C9190. J Clin Oncol. 2000 Jun; 18(12): 2444-58. [PubMed: 10856105]

25. Hauschild A, Weichenthal M, Rass K, et al. Efficacy of low-dose interferon \{alpha\}2a 18 versus 60 months of treatment in patients with primary melanoma of $>=1.5 \mathrm{~mm}$ tumor thickness: results of a randomized phase III DeCOG trial. J Clin Oncol. 2010 Feb 10; 28(5):841-6. [PubMed: 20048184]

26. Garbe C, Radny P, Linse R, et al. Adjuvant low-dose interferon \{alpha\}2a with or without dacarbazine compared with surgery alone: a prospective-randomized phase III DeCOG trial in melanoma patients with regional lymph node metastasis. Ann Oncol. 2008 Jun; 19(6):1195-201. [PubMed: 18281266]

27. Pehamberger H, Soyer HP, Steiner A, et al. Adjuvant interferon alfa-2a treatment in resected primary stage II cutaneous melanoma. Austrian Malignant Melanoma Cooperative Group. J Clin Oncol. 1998; 16(4):1425-1429. [PubMed: 9552047]

28. Grob JJ, Dreno B, de la Salmonière P, et al. Randomised trial of interferon alpha-2a as adjuvant therapy in resected primary melanoma thicker than $1.5 \mathrm{~mm}$ without clinically detectable node metastases. French Cooperative Group on Melanoma. Lancet. 1998; 351(9120):1905-1910. [PubMed: 9654256]

29. Eggermont AM, Suciu S, MacKie R, et al. Post-surgery adjuvant therapy with intermediate doses of interferon alfa $2 \mathrm{~b}$ versus observation in patients with stage IIb/III melanoma (EORTC 18952): randomised controlled trial. Lancet. 2005; 366(9492):1189-1196. [PubMed: 16198768]

30. Chiarion-Sileni V, Guida M, Romanini A, et al. for Italian Melanoma Intergroup (IMI). Intensified high-dose intravenous interferon alpha $2 \mathrm{~b}$ (IFNa2b) for adjuvant treatment of stage III melanoma: A randomized phase III Italian Melanoma Intergroup (IMI) trial [ISRCTN75125874]. J Clin Oncol. 2011; 29(suppl) abstr 8506.

31. Pectasides D, Dafni U, Bafaloukos D, et al. Randomized phase III study of 1 month versus 1 year of adjuvant high-dose interferon alfa- $2 \mathrm{~b}$ in patients with resected high-risk melanoma. J Clin Oncol. 2009 Feb 20; 27(6):939-44. [PubMed: 19139440]

32. Agarwala SS, Lee SJ, Flaherty LE, et al. Randomized phase III trial of high-dose interferon alfa- $2 b$ (HDI) for 4 weeks induction only in patients with intermediate- and high-risk melanoma (Intergroup trial E 1697). J Clin Oncol. 2011; 29(suppl) abstr 8505.

33. Kirkwood JM, Manola J, Ibrahim J, et al. for Eastern Cooperative Oncology Group. A pooled analysis of eastern cooperative oncology group and intergroup trials of adjuvant high-dose interferon for melanoma. Clin Cancer Res. 2004 Mar 1; 10(5):1670-7. [PubMed: 15014018] 
34. Bukowski R, Ernstoff MS, Gore ME, et al. Pegylated interferon alfa-2b treatment for patients with solid tumors: a phase I/II study. J Clin Oncol. 2002 Sep 15; 20(18):3841-9. [PubMed: 12228203]

35. Eggermont AM, Suciu S, Santinami M, et al. Adjuvant therapy with pegylated interferon alfa- $2 b$ versus observation alone in resected stage III melanoma: final results of EORTC 18991, a randomised phase III trial. Lancet. 2008; 372(9633):117-126. [PubMed: 18620949]

36. Eggermont AM, Suciu S, Testori A, et al. Ulceration and stage are predictive of interferon efficacy in melanoma: Results of the phase III adjuvant trials EORTC 18952 and EORTC 18991. Eur J Cancer. 2011 Nov 4.

37. Grob JJ, Jouary T, Dreno B, et al. Adjuvant therapy with pegylated interferon alfa-2b (36 months) versus low-dose interferon alfa- $2 \mathrm{~b}$ (18 months) in melanoma patients without macro-metastatic nodes: EADO trial. J Clin Oncol. 2010; 28(15 suppl) Abstract LBA8506.

38. Hansson J, Aamdal S, Bastholt L, et al. Two different durations of adjuvant therapy with intermediate-dose interferon alfa-2b in patients with high-risk melanoma (Nordic IFN trial): a randomised phase 3 trial. Lancet Oncol. 2011 Feb; 12(2):144-52. [PubMed: 21256809]

39. Morton, DL., et al. An international, randomized, phase III trial of bacillus Calmette-Guerin (BCG) plus allogeneic melanoma vaccine (MCV) or placebo after complete resection of melanoma metastatic to regional or distant sites. Presented at: 58th Annual Meeting of the Society of Surgical Oncology; Atlanta, GA, USA. 3-6 March 2006; Abstract 8508

40. Kirkwood JM, Ibrahim J, Lawson DH, et al. High-dose interferon alfa-2b does not diminish antibody response to GM2 vaccination in patients with resected melanoma: results of the Multicenter Eastern Cooperative Oncology Group Phase II Trial E2696. J Clin Oncol. 2001 Mar 1; 19(5):1430-6. [PubMed: 11230488]

41. Lawson DH, Lee SJ, Tarhini AA, et al. E4697: Phase III cooperative group study of yeast-derived granulocyte macrophage colony-stimulating factor (GM-CSF) versus placebo as adjuvant treatment of patients with completely resected stage III-IV melanoma. J Clin Oncol. 2010; 28(suppl):15s. abstr 8504.

42. Markovic S, Burch PA, LaPlant B, et al. Adjuvant GM-CSF therapy for patients with resected stage III/IV melanoma: A retrospective review of a single-center experience. J Clin Oncol. 2011; 29(suppl) abstr 8596.

43. Stuckert, JJ.; Tarhini, AA.; Lee, S., et al. Interferon alfa-induced autoimmunity and serum S100 levels as predictive and prognostic biomarkers in high-risk melanoma in the ECOG-intergroup phase II trial E2696. Journal of Clinical Oncology; ASCO Annual Meeting Proceedings Part I; 2007. p. 8506

44. Tarhini AA, Stuckert J, Lee S, et al. Prognostic significance of serum S100B protein in high-risk surgically resected melanoma patients participating in Intergroup Trial ECOG 1694. J Clin Oncol. 2009 Jan 1; 27(1):38-44. [PubMed: 19047287]

45. Gogas H, Ioannovich J, Dafni U, et al. Prognostic significance of autoimmunity during treatment of melanoma with interferon. N Engl J Med. 2006 Feb 16; 354(7):709-18. [PubMed: 16481638]

46. Bouwhuis MG, Suciu S, Testori A, et al. Phase III trial comparing adjuvant treatment with pegylated interferon Alfa-2b versus observation: prognostic significance of autoantibodies-EORTC 18991. J Clin Oncol. 2010 May 10; 28(14):2460-6. [PubMed: 20385998]

47. Davar D, Tarhini A, Kirkwood JM. Adjuvant Therapy: Melanoma. J Skin Cancer. 2011; 2011:19. Article ID 274382. 10.1155/2011/274382

48. Boni A, Cogdill AP, Dang P, et al. Selective BRAFV600E inhibition enhances T-cell recognition of melanoma without affecting lymphocyte function. Cancer Res. 2010 Jul 1; 70(13):5213-9. [PubMed: 20551059]

49. Livingston PO, Wong GY, Adluri S, et al. Improved survival in stage III melanoma patients with GM2 antibodies: a randomized trial of adjuvant vaccination with GM2 ganglioside. J Clin Oncol. 1994 May; 12(5):1036-44. [PubMed: 8164027]

50. Eggermont AM, Suciu S, Ruka W, et al. for EORTC Melanoma Group. EORTC 18961: Postoperative adjuvant ganglioside GM2-KLH21 vaccination treatment vs observation in stage II (T3T4NOM0) melanoma: 2nd interim analysis led to an early disclosure of the results. J Clin Oncol. 2008; 26(May 20 suppl) abstr 9004. 
51. Hersey P, Coates AS, McCarthy WH, et al. Adjuvant immunotherapy of patients with high-risk melanoma using vaccinia viral lysates of melanoma: results of a randomized trial. J Clin Oncol. 2002 Oct 15; 20(20):4181-90. [PubMed: 12377961]

52. Vantomme V, Boël P, De Plaen E, et al. A new tumor-specific antigenic peptide encoded by MAGE-6 is presented to cytolytic T lymphocytes by HLA-Cw16. Cancer Immun. 2003 Dec 10.3:17. [PubMed: 14664500]

53. Vantomme V, Dantinne C, Amrani N, et al. Immunologic analysis of a phase I/II study of vaccination with MAGE-3 protein combined with the AS02B adjuvant in patients with MAGE-3positive tumors. J Immunother. 2004 Mar-Apr;27(2):124-35. [PubMed: 14770084]

54. Morton DL, Hsueh EC, Essner R, et al. Prolonged survival of patients receiving active immunotherapy with Canvaxin therapeutic polyvalent vaccine after complete resection of melanoma metastatic to regional lymph nodes. Ann Surg. 2002 Oct; 236(4):438-48. discussion 448-9. [PubMed: 12368672]

55. Morton, DL.; Mozzillo, N.; Thompson, JF., et al. for the Malignant Melanoma Active Immunotherapy Trial 3 (MMAIT) investigators. An international, randomized, phase III trial of bacillus Calmette-Guerin (BCG) plus allogeneic melanoma vaccine (MCV) or placebo after complete resection of melanoma metastatic to regional or distant sites. Journal of Clinical Oncology; ASCO Annual Meeting Proceedings Part I; 2007. p. 8508

56. Kirkwood JM, Lorigan P, Hersey P, et al. Phase II trial of tremelimumab (CP-675,206) in patients with advanced refractory or relapsed melanoma. Clin Cancer Res. 2010 Feb 1; 16(3):1042-8. [PubMed: 20086001]

57. Ribas A, Hauschild A, Kefford R, et al. Phase III, open-label, randomized, comparative study of tremelimumab (CP-675,206) and chemotherapy (temozolomide [TMZ] or dacarbazine [DTIC]) in patients with advanced melanoma. J Clin Oncol. 2008; 26(May 20 suppl) abstr LBA9011.

58. Hodi FS, O'Day SJ, McDermott DF, et al. Improved survival with ipilimumab in patients with metastatic melanoma. N Engl J Med. 2010 Aug 19; 363(8):711-23. [PubMed: 20525992]

59. Lebbe C, Hoos A, Chin K, et al. Effect of Dose on Efficacy and Safety in Ipilimumab-treated Patients with Advanced Melanoma: Results from a Phase II, Randomized, Dose-ranging Study. European Society for Medical Oncology. 2008 Oral Presentation No. 769015 September 2008. (Study 022).

60. Wolchok JD, Thomas L, Bondarenko IN, et al. Phase III randomized study of ipilimumab (IPI) plus dacarbazine (DTIC) versus DTIC alone as first-line treatment in patients with unresectable stage III or IV melanoma. J Clin Oncol. 2011; 29(suppl) abstr LBA5.

61. Robert C, Thomas L, Bondarenko IN, et al. Ipilimumab plus dacarbazine for previously untreated metastatic melanoma. N Engl J Med. 2011 Jun 30; 364(26):2517-26. [PubMed: 21639810]

62. Hamid O, Chasalow SD, Tsuchihashi Z, et al. Association of baseline and on study tumor biopsy markers with clinical activity in patients (pts) with advanced melanoma treated with ipilimumab. J Clin Oncol. 2009; 27(suppl):15s. abstr 9008.

63. Wang W, Lau R, Yu D, et al. PD1 blockade reverses the suppression of melanoma antigen-specific CTL by CD4+CD25Hi regulatory T cells. Int Immunol. 2009; 21(9):1065-1077. [PubMed: 19651643]

64. DeVita, VT.; Lawrence, TS.; Rosenberg, SA. DeVita, Hellman, and Rosenberg's Cancer: Principles \& Practice of Oncology. Lippincott Williams \& Wilkins; 2008. p. 1929-1930.

65. Agarwala SS, Neuberg D, Park Y, et al. Mature results of a phase III randomized trial of bacillus Calmette-Guerin (BCG) versus observation and BCG plus dacarbazine versus BCG in the adjuvant therapy of American Joint Committee on Cancer Stage I-III melanoma (E1673): a trial of the Eastern Oncology Group. Cancer. 2004 Apr 15; 100(8):1692-8. [PubMed: 15073858]

66. Garbe C, Radny P, Linse R, et al. Adjuvant low-dose interferon \{alpha\} 2a with or without dacarbazine compared with surgery alone: a prospective-randomized phase III DeCOG trial in melanoma patients with regional lymph node metastasis. Ann Oncol. 2008 Jun; 19(6):1195-2011. [PubMed: 18281266]

67. Chang DT, Amdur RJ, Morris CG, et al. Adjuvant radiotherapy for cutaneous melanoma: comparing hypofractionation to conventional fractionation. Int J Radiat Oncol Biol Phys. 2006 Nov 15; 66(4):1051-5. [PubMed: 16973303] 
68. Henderson MA, Burmeister B, Thompson JF, et al. Adjuvant radiotherapy and regional lymph node field control in melanoma patients after lymphadenectomy: Results of an intergroup randomized trial (ANZMTG 01.02/TROG 02.01). J Clin Oncol. 2009; 27(suppl):18s. abstr LBA9084.

69. Weber JS, Yu B, Hall M, et al. Pharmacodynamic and predictive markers of ipilimumab on melanoma patients' T-cells. J Clin Oncol. 2011; 29(suppl) abstr 2503.

70. Tarhini AA, Edington H, Butterfield LH, et al. Neoadjuvant ipilimumab in patients with stage IIIB/ C melanoma: Immunogenicity and biomarker analysis. J Clin Oncol. 2011; 29(suppl) abstr 8536.

71. Sarnaik AA, Yu B, Yu D, et al. Extended dose ipilimumab with a peptide vaccine: immune correlates associated with clinical benefit in patients with resected high-risk stage IIIc/IV melanoma. Clin Cancer Res. 2011 Feb 15; 17(4):896-906. [PubMed: 21106722]

72. Dematteo RP, Ballman KV, Antonescu CR, et al. Adjuvant imatinib mesylate after resection of localised, primary gastrointestinal stromal tumour: a randomised, double-blind, placebo-controlled trial. Lancet. 2009 Mar 28; 373(9669):1097-104. [PubMed: 19303137]

73. Joensuu, H.; Eriksson, M.; Hartmann, J., et al. Twelve vs 36 months of adjuvant imatinib as treatment of operable GIST with a high risk of recurrence: Final results of a randomized trial (SSGXVIII/AIO). ASCO Annual Meeting; 2011. Abstract LBA1 


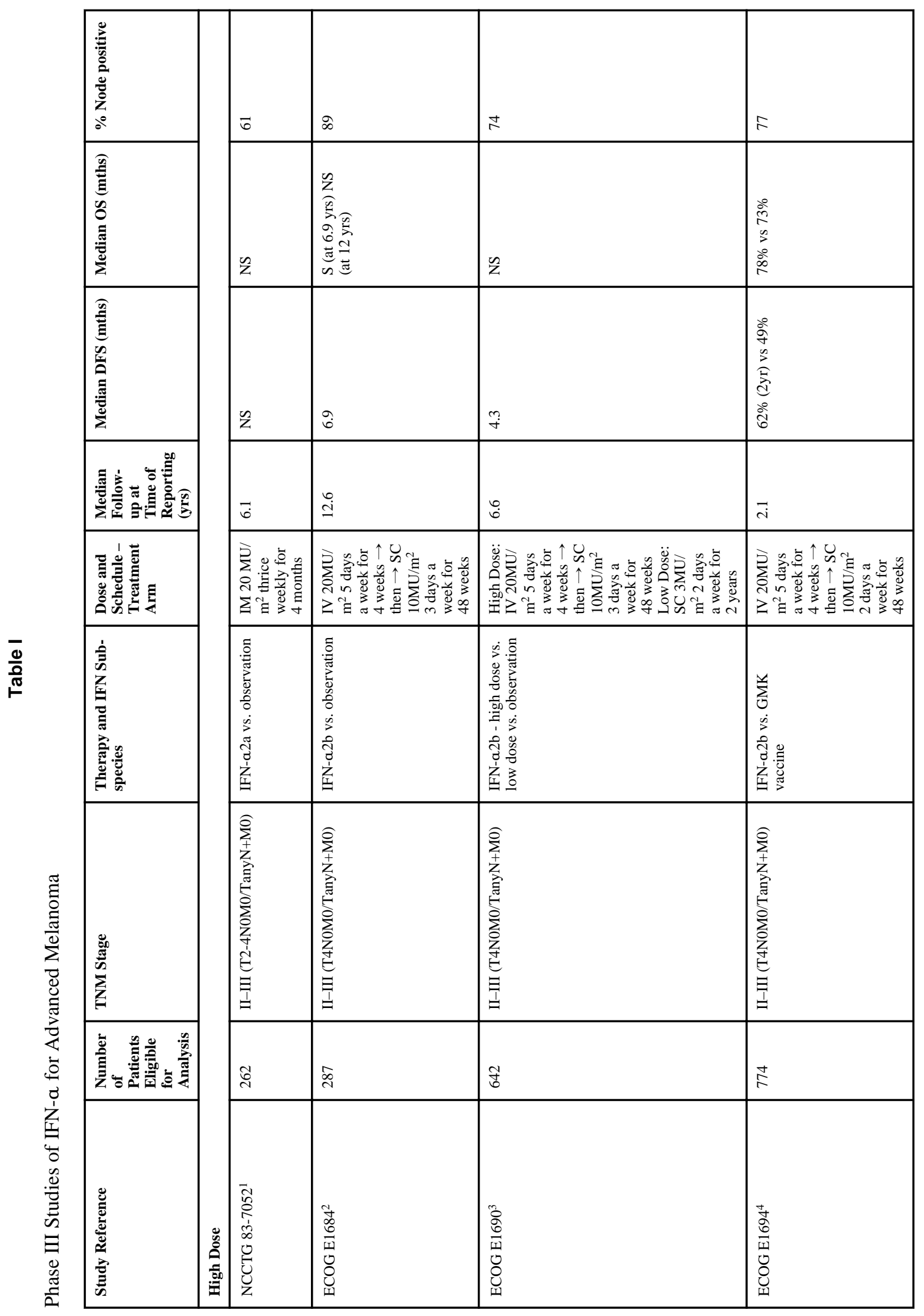






Cancer J. Author manuscript; available in PMC 2013 March 01. 


\begin{tabular}{|c|c|c|c|c|c|c|c|c|}
\hline 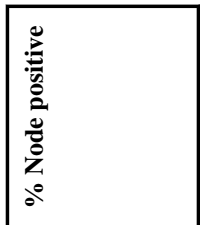 & & 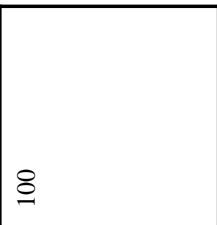 & 0 & 0 & 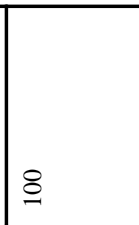 & 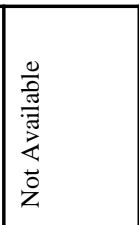 & $\infty$ & 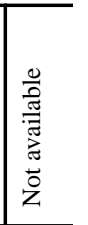 \\
\hline 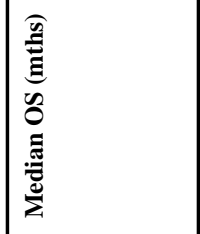 & & $\tilde{z}$ & 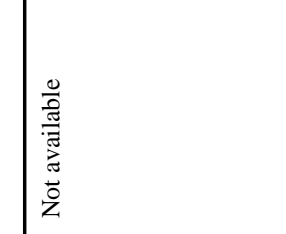 & 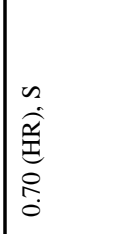 & $n^{n}$ & $\tilde{z}$ & $\tilde{z}$ & $\eta$ \\
\hline 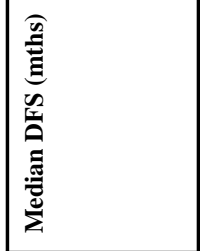 & & 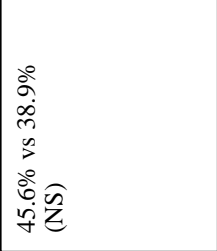 & $\infty$ & $\mid$ & $\mathrm{n}$ & $\mathrm{n}$ & $\frac{n}{z}$ & $\tilde{z}$ \\
\hline 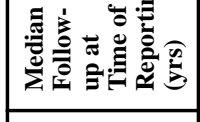 & & 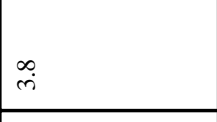 & $\stackrel{+}{m}$ & $\lambda$ & $?$ & $\stackrel{0}{\circ}$ & $\begin{array}{l}\infty \\
\infty \\
\end{array}$ & $\vec{m}$ \\
\hline 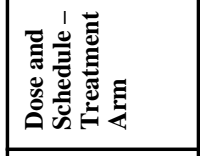 & 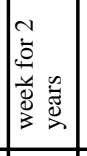 & 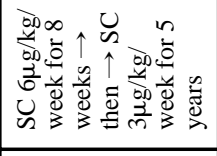 & 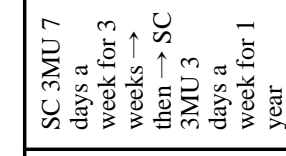 & 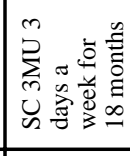 & 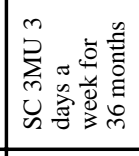 & 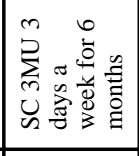 & 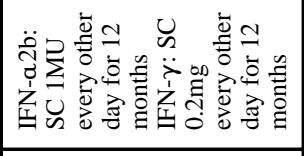 & 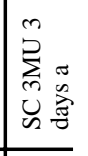 \\
\hline 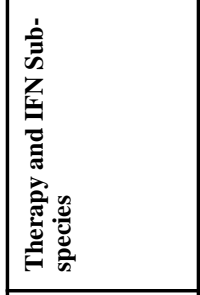 & & 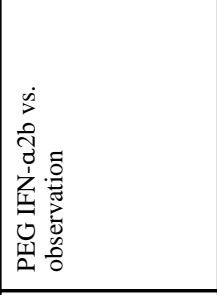 & 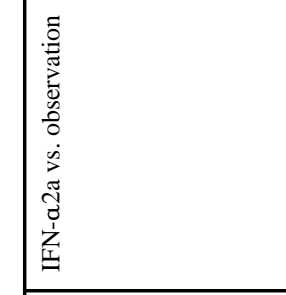 & 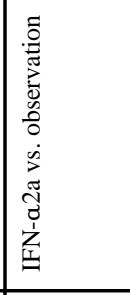 & 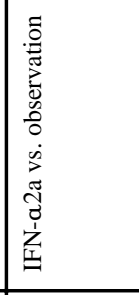 & 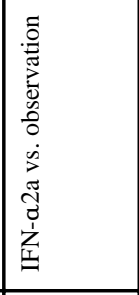 & 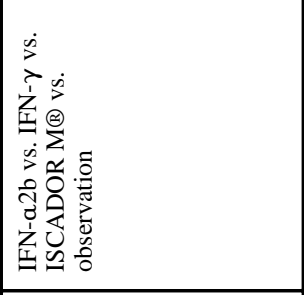 & 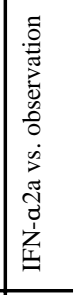 \\
\hline 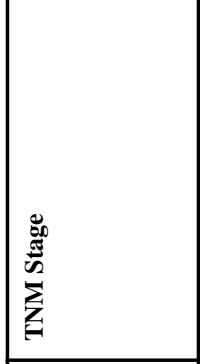 & & 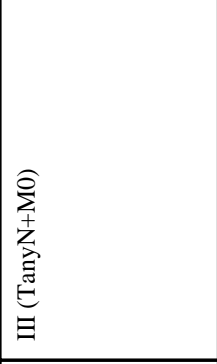 & 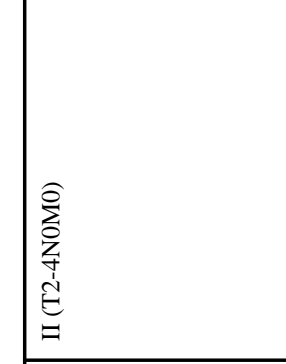 & 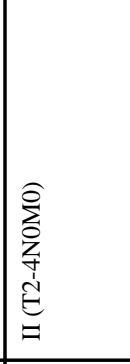 & 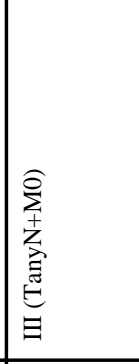 & 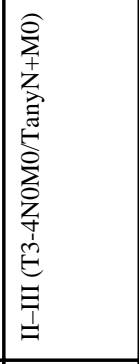 & 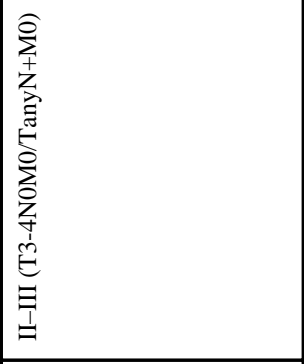 & 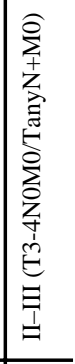 \\
\hline 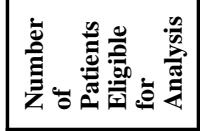 & & đั & $\bar{m}$ & 票 & 表 & 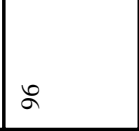 & 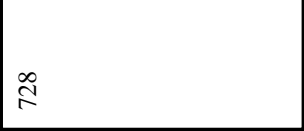 & 茍 \\
\hline 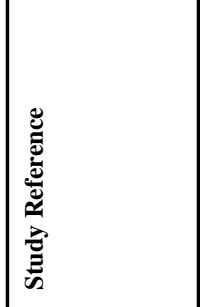 & & $\begin{array}{l}\infty \\
\bar{\alpha} \\
0 \\
0 \\
0 \\
0 \\
0 \\
0 \\
0\end{array}$ & 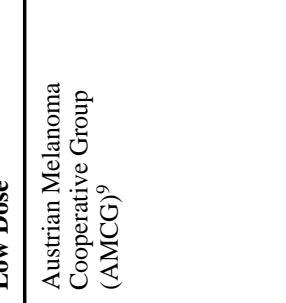 & 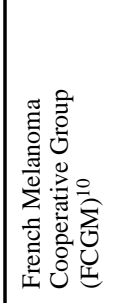 & 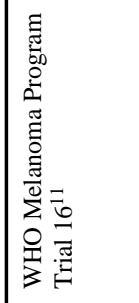 & 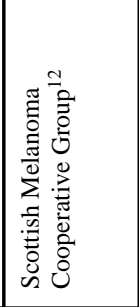 & 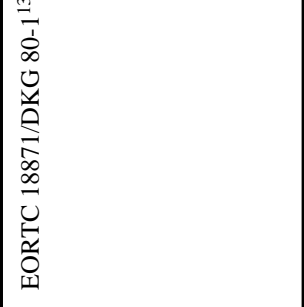 & 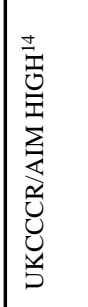 \\
\hline
\end{tabular}

Cancer J. Author manuscript; available in PMC 2013 March 01. 


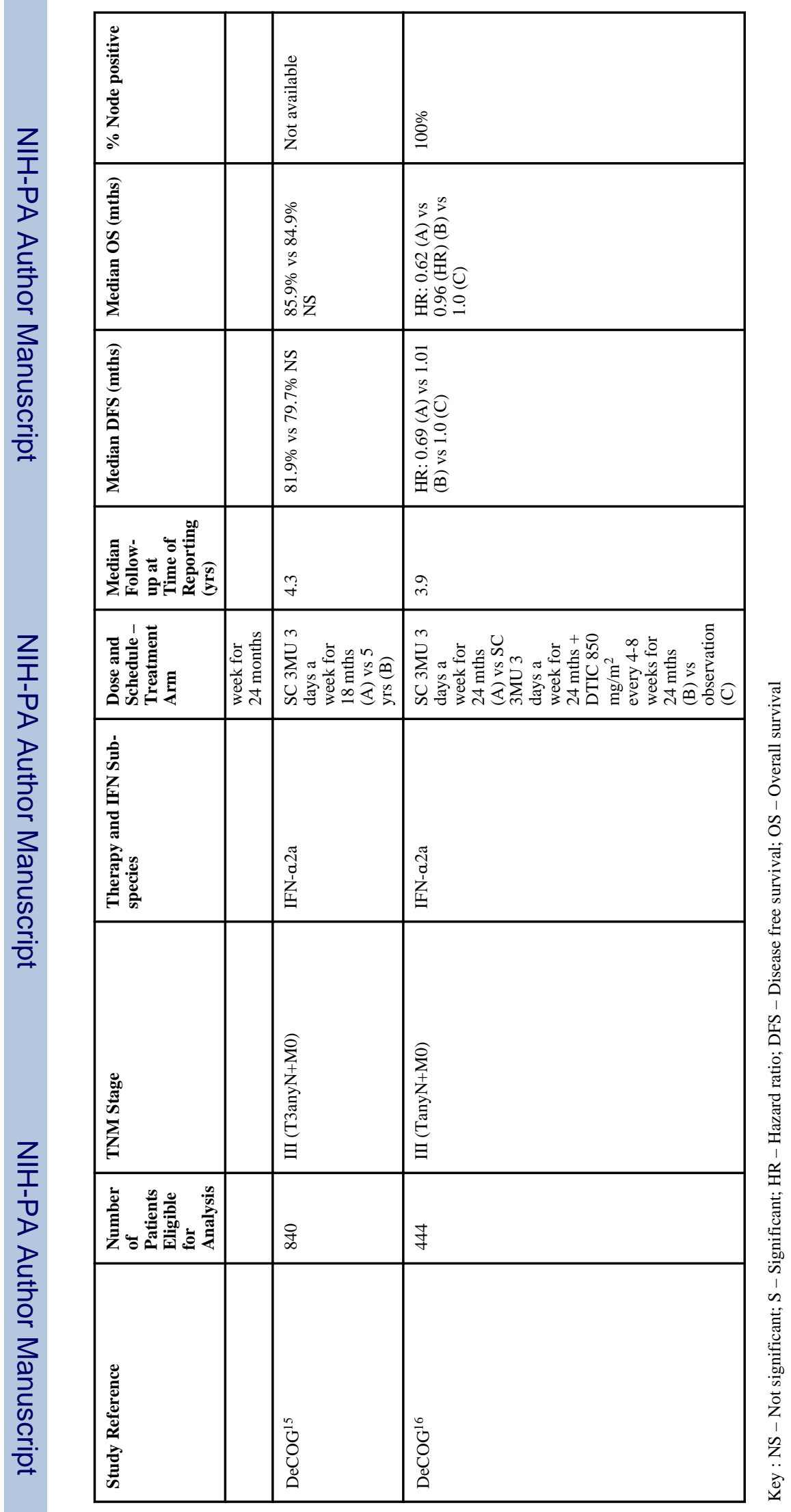

Cancer J. Author manuscript; available in PMC 2013 March 01. 


\begin{tabular}{|c|c|c|c|c|c|c|c|}
\hline 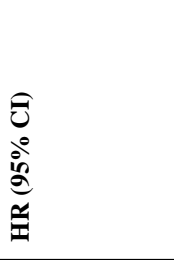 & $\overleftrightarrow{z}$ & $\frac{\pi}{z}$ & $\overleftrightarrow{z}$ & 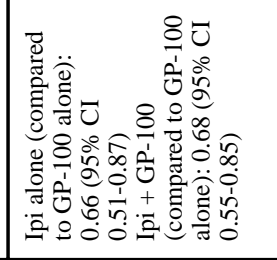 & 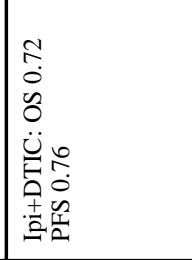 & 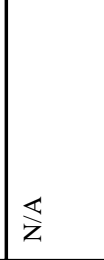 & 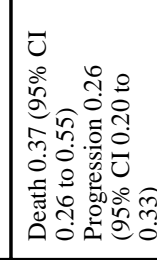 \\
\hline 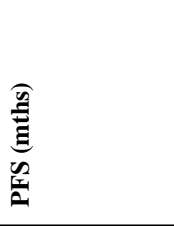 & $\overleftrightarrow{z}$ & $\underline{\underline{z}}$ & $\underline{\Sigma}$ & 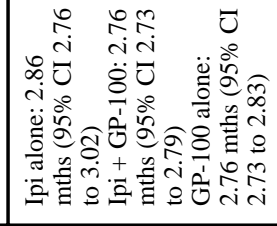 & 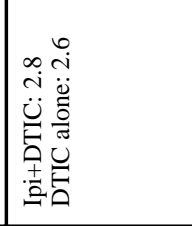 & : & 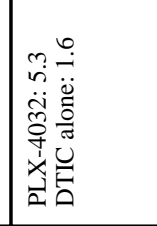 \\
\hline 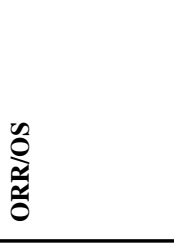 & 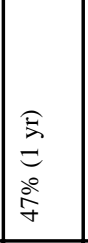 & 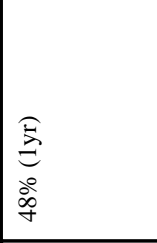 & 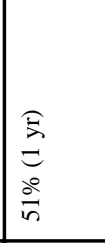 & 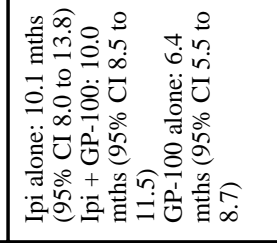 & 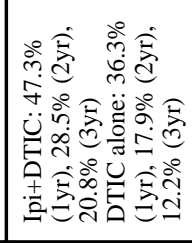 & 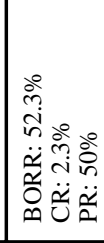 & 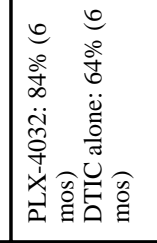 \\
\hline 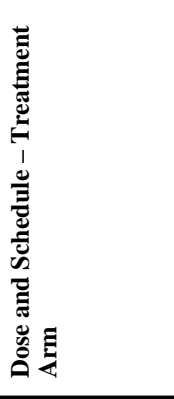 & 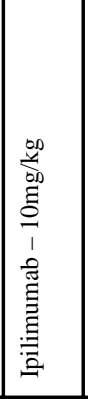 & 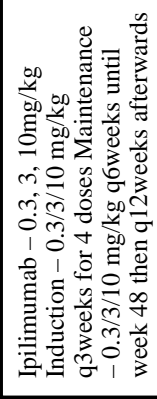 & 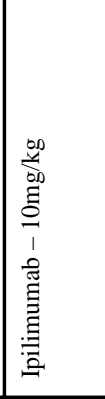 & 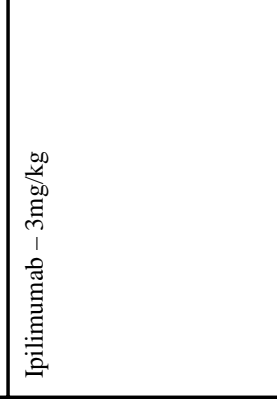 &  & 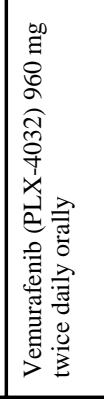 & 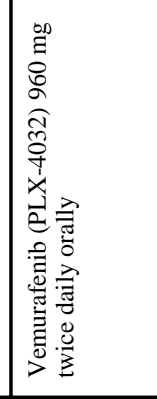 \\
\hline  &  & 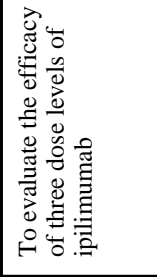 & 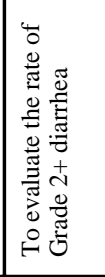 & 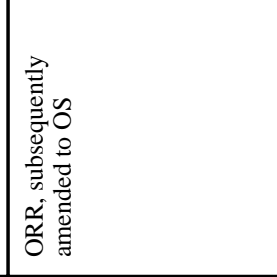 & 8 & $\mid$ & o \\
\hline  & 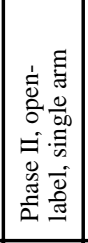 &  & 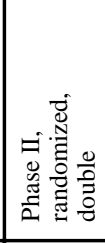 & 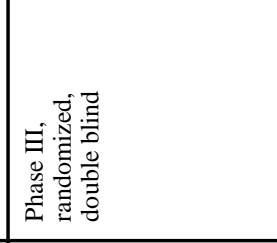 & 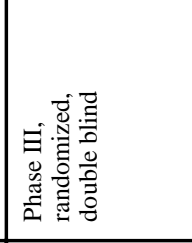 & 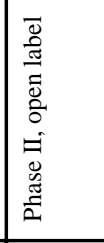 & 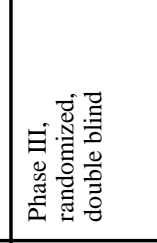 \\
\hline 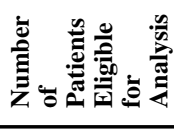 & $\stackrel{2}{2}$ & $\overrightarrow{\mathrm{A}}$ & $\stackrel{n}{=}$ & 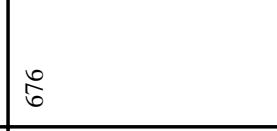 & 总 & 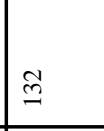 & 16 \\
\hline 䓀 & \begin{tabular}{|l|}
$\infty$ \\
$\infty$ \\
0 \\
$c$ \\
$\sum_{n}^{n}$ \\
\end{tabular} & 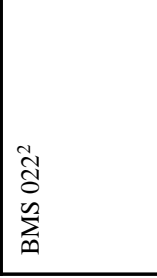 & 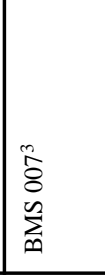 & 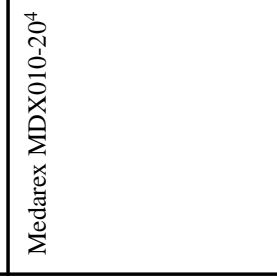 & 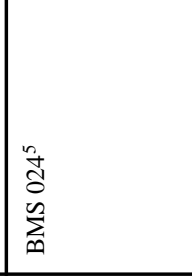 & $\sum_{\substack{\infty\\
}}^{\stackrel{\infty}{N}}$ & $\sum_{\text {兽 }}^{\infty}$ \\
\hline
\end{tabular}




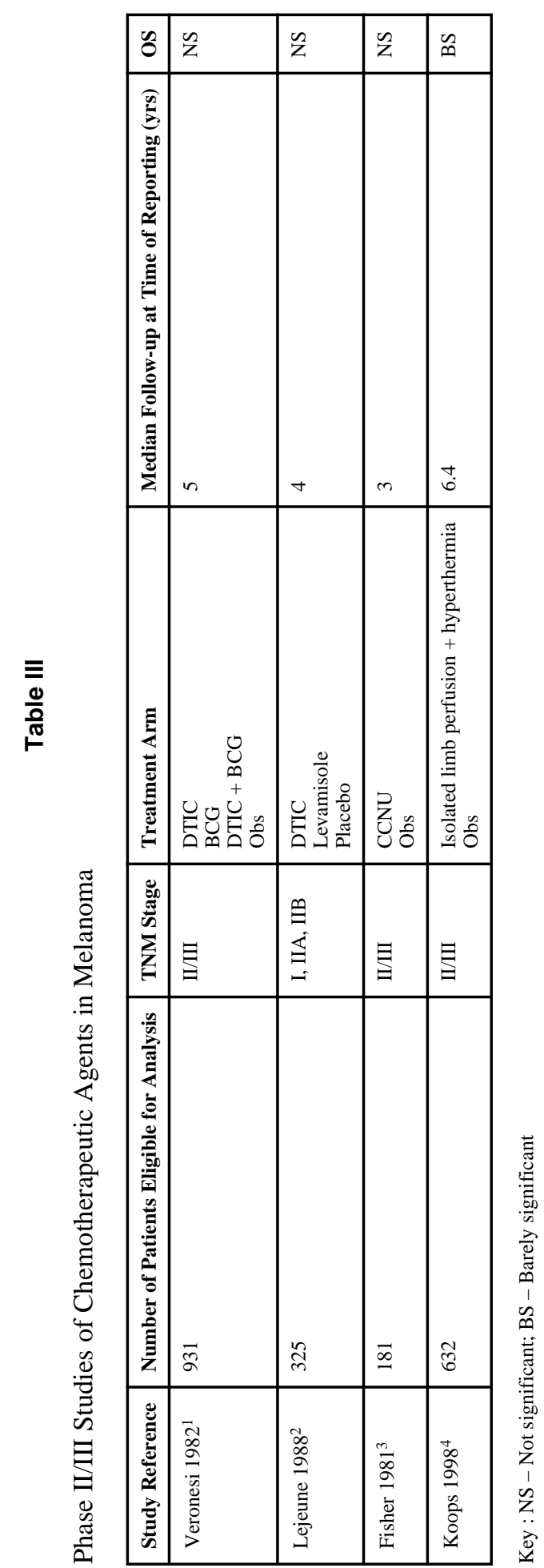

Cancer J. Author manuscript; available in PMC 2013 March 01. 